\title{
Supergravity solutions from floating branes
}

\author{
losif Bena, ${ }^{a}$ Stefano Giusto, ${ }^{a}$ Clément Ruef $^{a}$ and Nicholas P. Warner ${ }^{b}$ \\ ${ }^{a}$ Institut de Physique Théorique, \\ CEA Saclay, 91191 Gif sur Yvette, France \\ ${ }^{b}$ Department of Physics and Astronomy, University of Southern California, \\ Los Angeles, CA 90089, U.S.A. \\ E-mail: iosif.bena@cea.fr, stefano.giusto@cea.fr, clement.ruef@cea.fr, \\ warner@usc . edu
}

ABSTRACT: We simplify the equations of motion of five-dimensional ungauged supergravity coupled to three U(1) gauge fields using a floating-brane Ansatz in which the electric potentials are directly related to the gravitational warp factors. We find a new class of nonBPS solutions, that can be obtained linearly starting from an Euclidean four-dimensional Einstein-Maxwell base. This class - the largest known so far - reduces to the BPS and almost-BPS solutions in certain limits. We solve the equations explicitly when the base space is given by the Israel-Wilson metric, and obtain solutions describing non-BPS D6 and anti-D6 branes kept in equilibrium by flux. We also examine the action of spectral flow on solutions with an Israel-Wilson base and show that it relates these solutions to almost-BPS solutions with a Gibbons-Hawking base.

KeYwords: Black Holes in String Theory, D-branes 


\section{Contents}

1 Introduction 1

2 Equations of motion $\quad 4$

2.1 Conventions and the floating-brane Ansatz 4

2.2 Einstein's equations $\quad 6$

$\begin{array}{lll}2.3 & \text { Scalar equations } & 6\end{array}$

$\begin{array}{ll}2.4 \text { Maxwell equations } & 7\end{array}$

$\begin{array}{lll}3 & \text { Solving the system } & 7\end{array}$

$\begin{array}{lll}3.1 & \text { General results } & 7\end{array}$

3.2 A simple assumption 8

$\begin{array}{ll}3.3 & \text { A further assumption and a linear system }\end{array}$

4 Israel-Wilson metrics $\quad 10$

$\begin{array}{ll}\text { 4.1 The Israel-Wilson background } & 10\end{array}$

4.2 Harmonic forms 11

$\begin{array}{ll}4.3 & \text { The linear system } \\ & 12\end{array}$

4.4 An explicit example: a non-BPS black hole in an Israel-Wilson metric 13

4.5 The BPS and almost-BPS limits of solutions with an Israel-Wilson base 15

5 Spectral flow and the Israel-Wilson metric $\quad \mathbf{1 5}$

$\begin{array}{ll}5.1 \text { The D1-D5-P duality frame } & 16\end{array}$

$\begin{array}{ll}5.2 \text { The action of spectral flow } & 17\end{array}$

$\begin{array}{ll}5.3 \text { Spectral flow and smooth horizonless multi-center solutions. } & 19\end{array}$

6 Conclusion $\quad 20$

\section{Introduction}

The classification of supergravity solutions is an important program that has yielded an amazing amount of new physics and, in particular, has greatly enhanced our understanding of the AdS-CFT correspondence, the non-perturbative dynamics of string theory, and the physics of black holes. In pure gravity, there has been extensive work on classifying fourdimensional solutions with horizons and, in higher dimensions, there has been extensive work on systems with enough Killing symmetries to reduce to a two-dimensional problem that can be solved using integrability (see, for example, [1]).

In supergravity and string theory most of the classification work has focussed on supersymmetric solutions, and is done essentially by using Killing spinors or $G$-structures to reduce the second-order supergravity equations of motion to first-order equations (see, for 
example, $[2-5])$. It is clearly important to extend this work to non-supersymmetric solutions, not only because we would like to better understand non-supersymmetric physics, but also because we expect (from the dynamics of string theory probes) to find rather large classes of non-supersymmetric solutions, with very interesting properties. The problem is, of course, to solve the requisite non-linear partial differential equations. If the system has enough symmetry to reduce it to an effective two-dimensional system then the equations of motion often become those of an integrable system and thus substantial progress can be, and has been, made (see, for example, [6-13]). To make progress more generally one must incorporate some of the physics that one wishes to solve so as to provide a guiding structure that enables one to solve the equations. One promising new approach is to use "black-folds" $[14,15]$ in which one rewrites the equations of motion in terms of intrinsic, p-brane equations and extrinsic equations that describe the embedding. For time-independent solutions, the intrinsic p-brane equations can be solved explicitly and this gives valuable insight into the structure of the overall solution. A summary of recent results on higher-dimensional black holes is found in [16].

One of our purposes in this paper is to provide a rather different, physically motivated guiding structure for finding interesting, non-supersymmetric solutions of five dimensional $\mathrm{U}(1)^{3}$ ungauged supergravity. These solutions uplift to solutions of eleven-dimensional supergravity on a six-torus, and when compactified to fourdimensions correspond to solutions of the STU model. In the Ansatz we use, the warp factors and the electric potentials are equal and hence the probe M2 branes that have the same charge vector as the solution feel no force. Therefore, we will call this the floating brane Ansatz.

This Ansatz naturally incorporates the known BPS [17-19] and almost-BPS [20] solutions of five-dimensional ungauged supergravity, ${ }^{1}$ but, as we will see, the equations governing the general floating-brane solutions are much more general. The mass of these solutions depends linearly on their M2 charges (this comes from the equality of the warp factors and electric potential) and thus many of the floating-brane solutions will be extremal, but there are also some interesting (but rather restrictive) classes of non-extremal floating-brane solutions [23, 25].

The purpose of this paper is four-fold. First, we examine the full supergravity equations of motion using the floating-brane Ansatz, and show how to obtain the usual BPS and almost-BPS solutions. In addition, we find that the linear equations governing these solutions lead to solutions to the supergravity equations of motion not only when the base space is hyper-Kähler, but also when the base space is merely an arbitrary four-dimensional Ricci-flat manifold. ${ }^{2}$ In hindsight one should have expected this - after all, having a complex structure on the base is needed for supersymmetry, but Einstein's equations only see the Ricci tensor of the base.

Secondly, within the floating-brane Ansatz, we find, after a few simplifying assumptions, a new class of solutions that solve the five-dimensional supergravity equations of motion. The equations governing these simplified-floating-brane solutions can still be solved

\footnotetext{
${ }^{1}$ For a few recent examples of almost-BPS solutions see [21-24].

${ }^{2}$ Examples of such solutions that have Euclidean Schwarzschild and Kerr-Taub-Bolt base spaces are explicitly constructed in [23].
} 
in a linear fashion, but they are more general than both the BPS and the almost-BPS equations, and reduce to these in certain limits. In particular, the simplified-floating-brane solutions have a four-dimensional base space that does not need to be Ricci-flat. Rather, to construct these solutions the starting point is a four-dimensional Euclidean "electrovac" solution of the Einstein-Maxwell equations. Given such a solution one can turn on two-form field strengths on various two-cycles, and solve a set of coupled linear equations to find the remaining two Maxwell field strengths and two of the warp factors. One then solves another set of coupled linear equations to find the rotation vector and the remaining warp factor. Hence, one can construct full non-BPS solutions of the five-dimensional supergravity equations of motion starting from any four-dimensional Euclidean electrovac solution, and solving only linear equations.

The third purpose of this paper is to illustrate the method outlined above and to construct simplified-floating-brane solutions using Israel-Wilson geometries as base spaces. These geometries are a special class of non-Ricci-flat electrovac solutions that have a U(1) isometry. The new equations imply that the functions determining the magnetic field strengths are no longer harmonic in the $\mathbb{R}^{3}$ base of the Israel-Wilson space, but satisfy a linear system of coupled differential equations that relate them to some of the warp factors. We solve this system for the particular example of an Israel-Wilson base whose fiber degenerates at two locations, and obtain a solution that, in a certain limit, reduces to a BPS black hole in Taub-NUT, and in a different limit reduces to a non-BPS black ring in Taub-NUT. ${ }^{3}$ This solution also has a two-cycle with non-trivial flux, and one can use it to obtain a very large class of smooth horizonless solutions.

The fourth purpose of this paper is to relate the solutions in our new class that are constructed using an asymptotically $\mathbb{R}^{3} \times S^{1}$ Israel-Wilson base space to the known multicenter almost-BPS solutions in Taub-NUT [21, 22]. We find that the two classes of solutions can be transformed into each other upon applying the "spectral flow" transformation of supergravity solutions with a U(1) isometry discussed in [27]. From the perspective of sixdimensional supergravity (or of the full solution written in a IIB duality frame where the M2 charges correspond to D1, D5, and P charges) this transformation mixes the KaluzaKlein ${ }^{4} \mathrm{U}(1)$ and the $\mathrm{U}(1)$ of the base. For BPS solutions, this spectral flow transformation re-shuffles the D6, D4, D2 and D0 charges and moduli, but the resulting solution is still BPS and hence remains in the class of solutions of [17-19]. However, when applying this spectral flow transformation to an almost-BPS solution, the resulting solution is no longer an almost-BPS solution, but is a simplified-floating-brane solution with an Israel-Wilson base space.

An immediate corollary of this observation is that among within the floating-brane solutions there exist not only multiple black holes, but also new smooth horizonless bubbling

\footnotetext{
${ }^{3}$ An extremal non -BPS black ring solution in Taub-NUT was also found in [26]. That solution is presented in a duality frame different from the one we use in this paper, in which the asymptotic charges are D0 and D6. We note that the 3D base metric of the solution in [26] is not conformally flat, while our non-BPS black ring in Taub-NUT has a flat 3D base. Since dualities can only change the 3D metric up to a conformal factor, the two solutions cannot be duality equivalent.

${ }^{4}$ That is, the U(1) common to the D1 and D5 branes.
} 
solutions, that have non-trivial magnetic fluxes on the two-cycles of the Israel-Wilson base. Recall that this was not possible for the almost-BPS solutions: The anti-self-dual flux on the two-cycles of a multi-center Taub-NUT space is non-normalizable, and does not lead to asymptotically-flat solutions. Given that solutions in our new class can be obtained by spectral flow from almost-BPS solutions, it is straightforward to obtain smooth solutions with non-trivial fluxes by spectrally-flowing multiple supertubes.

In section 2 we begin by specifying the floating-brane Ansatz and then deriving the equations of motion. For simplicity we work with the ungauged five-dimensional supergravity action with three $\mathrm{U}(1)$ gauge fields (or the STU model upon reduction to fourdimensions). It would be interesting to see how difficult it is to extend our solutions to more general ungauged $\mathrm{U}(1)^{N}$ supergravities. In section 3 we first solve the system of equations as far as possible without making any additional assumptions beyond the original Ansatz. We then we make some simple assumptions that lead to a new linear system of equations that yield new classes of "simplified-floating-brane" non-BPS solutions that have as a base space a four-dimensional Einstein-Maxwell Euclidean electrovac solution. These equations, together with the recipe for constructing simplified-floating-brane solutions are given in sub-section 3.3. In section 4 we solve this linear system of equations when the base space is an Israel-Wilson metric, and present an explicit solution describing a non-BPS D6-D4-D2-D0 black hole in an anti-D6 background. In section 5 we discuss the effect of spectral flow on the solutions we build, and show that the simplified-floating-brane solutions with an Israel-Wilson base can be mapped to almost-BPS solutions that have a Gibbons-Hawking base space. In section 6 we conclude and outline future directions.

\section{Equations of motion}

\subsection{Conventions and the floating-brane Ansatz}

We consider $\mathcal{N}=2$, five-dimensional supergravity with three $\mathrm{U}(1)$ gauged fields and we use the conventions of [20]. The bosonic action is: ${ }^{5}$

$$
S=\frac{1}{2 \kappa_{5}} \int \sqrt{-g} d^{5} x\left(R-\frac{1}{2} Q_{I J} F_{\mu \nu}^{I} F^{J \mu \nu}-Q_{I J} \partial_{\mu} X^{I} \partial^{\mu} X^{J}-\frac{1}{24} C_{I J K} F_{\mu \nu}^{I} F_{\rho \sigma}^{J} A_{\lambda}^{K} \bar{\epsilon}^{\mu \nu \rho \sigma \lambda}\right),
$$

with $I, J=1,2,3$. One of the photons lies in the gravity multiplet and so there are only two vector multiplets and hence only two independent scalars. Thus the scalars, $X^{I}$, satisfy a constraint, and it is convenient to introduce three other scalar fields, $Z_{I}$, to parametrize these two scalars:

$$
X^{1} X^{2} X^{3}=1, \quad X^{1}=\left(\frac{Z_{2} Z_{3}}{Z_{1}^{2}}\right)^{1 / 3}, \quad X^{2}=\left(\frac{Z_{1} Z_{3}}{Z_{2}^{2}}\right)^{1 / 3}, \quad X^{3}=\left(\frac{Z_{1} Z_{2}}{Z_{3}^{2}}\right)^{1 / 3} .
$$

The scalar kinetic term can be written as:

$$
Q_{I J}=\frac{1}{2} \operatorname{diag}\left(\left(X^{1}\right)^{-2},\left(X^{2}\right)^{-2},\left(X^{3}\right)^{-2}\right) .
$$

\footnotetext{
${ }^{5}$ We take $\sqrt{-g} \bar{\epsilon}^{01234}=-1$.
} 
Note that the scalars, $X^{I}$, only depend upon the ratios $Z_{J} / Z_{K}$ and it is convenient to parametrize the third independent scalar by:

$$
Z \equiv\left(Z_{1} Z_{2} Z_{3}\right)^{1 / 3}
$$

We now use the scalar, $Z$, in the metric Ansatz:

$$
d s_{5}^{2}=-Z^{-2}(d t+k)^{2}+Z d s_{4}^{2},
$$

where the powers guarantee that $Z$ becomes an independent scalar from the fourdimensional perspective. We will denote the frames for (2.5) by $e^{A}, A=0,1, \ldots, 4$ and let $\hat{e}^{a}, a=1, \ldots, 4$ denote frames for $d s_{4}^{2}$. That is, we take:

$$
e^{0} \equiv-Z^{-1}(d t+k), \quad e^{a} \equiv Z^{1 / 2} \hat{e}^{a} .
$$

The heart of the "floating brane" Ansatz is to relate the metric coefficients and the scalars to the electrostatic potentials. The Maxwell Ansatz is thus:

$$
A^{(I)}=-\varepsilon Z_{I}^{-1}(d t+k)+B^{(I)},
$$

where $B^{(I)}$ is a one-form on the base (with metric $d s_{4}^{2}$ ). The parameter, $\varepsilon$, will be related to the self-duality or anti-self-duality of the fields in the solution and is fixed to have $\varepsilon^{2}=1$. Upon uplifting this solutions to eleven-dimensional supergravity, or M-theory, this Ansatz implies that M2 brane probes that have the same charge vector as the M2 charge vector of the solution will have equal and opposite Wess-Zumino and Born-Infeld terms and hence will feel no force. Such brane probes may be placed anywhere in the base and may thus be viewed as "floating."

It is convenient to define the field strengths:

$$
\Theta^{(I)} \equiv d B^{(I)}=\frac{1}{2} Z^{-1} \Theta_{a b}^{(I)} e^{a} \wedge e^{b}=\frac{1}{2} \Theta_{a b}^{(I)} \hat{e}^{a} \wedge \hat{e}^{b} .
$$

and

$$
K \equiv d k=\frac{1}{2}\left(\partial_{\mu} k_{\nu}-\partial_{\nu} k_{\mu}\right) d x^{\mu} \wedge d x^{\mu}=\frac{1}{2} K_{a b} \hat{e}^{a} \wedge \hat{e}^{b} .
$$

Note that the frame components are defined relative to the frames on $d s_{4}^{2}$.

Another consequence of the fact that we have used the same function, $Z$, in both the metric and the electric potential in (2.7) is that the mass of our solutions will always be linear in the electric (M2) charges, much like the mass of extremal solutions (although for some orientations the mass may also decrease linearly with the charges [23]). This also suggests that our solutions should be essentially extremal, however we have made no assumptions about the base metric, $d s_{4}^{2}$, and the choices for this will lead to a very large class of non-BPS solutions that include non-extremal solutions. 


\subsection{Einstein's equations}

The time (00) components of Einstein's equations give:

$$
\sum_{I} Z_{I}^{-1} \hat{\nabla}^{2} Z_{I}=-\frac{1}{4} Z^{-3} \sum_{I} Z_{I} \Theta_{a b}^{(I)}\left(Z_{I} \Theta_{a b}^{(I)}-2 \varepsilon K_{a b}\right)
$$

where $\hat{\nabla}$ is the covariant derivative in the base metric, $d s_{4}^{2}$.

The off-diagonal ( $0 a$ components) of Einstein's equations give:

$$
\hat{\nabla}^{b} K_{b a}=\varepsilon \sum_{I}\left(\hat{\nabla}^{b} Z_{I}\right) \Theta_{b a}^{(I)}
$$

or, equivalently,

$$
d *_{4} K=\varepsilon \sum_{I} d Z_{I} \wedge *_{4} \Theta^{(I)} .
$$

To give the remaining Einstein's equations it is convenient to define the two-form:

$$
\mathcal{P} \equiv K-\frac{1}{2} \varepsilon \sum_{I=1}^{3} Z_{I} \Theta^{(I)} .
$$

The components of Einstein's equations on the four-dimensional base are:

$$
\begin{aligned}
\hat{R}_{a b}-\frac{1}{2} \hat{R} \delta_{a b}=Z^{-3}[ & \mathcal{P}_{a c} \mathcal{P}_{b c}-\frac{1}{4} \delta_{a b} \mathcal{P}_{c d} \mathcal{P}_{c d} \\
& +\frac{1}{4}\left(2 \sum_{I} Z_{I}^{2} \Theta_{a c}^{(I)} \Theta_{b c}^{(I)}-\sum_{I, J} Z_{I} Z_{J} \Theta_{a c}^{(I)} \Theta_{b c}^{(J)}\right) \\
& \left.\quad-\frac{1}{16} \delta_{a b}\left(2 \sum_{I} Z_{I}^{2} \Theta_{c d}^{(I)} \Theta_{c d}^{(I)}-\sum_{I, J} Z_{I} Z_{J} \Theta_{c d}^{(I)} \Theta_{c d}^{(J)}\right)\right],
\end{aligned}
$$

where $\hat{R}_{a b}$ and $\hat{R}$ are the Ricci tensor and Ricci scalar of the base metric, $d s_{4}^{2}$. Note that these equations imply that the Ricci scalar of the base must vanish:

$$
\hat{R}=0 \text {. }
$$

\subsection{Scalar equations}

The scalar equations of motion yield equations for the ratios of the $Z_{I}$. For example:

$$
\begin{aligned}
Z_{1}^{-1} \hat{\nabla}^{2} Z_{1}-Z_{3}^{-1} \hat{\nabla}^{2} Z_{3}=\frac{1}{2} Z^{-3}[ & Z_{1} \Theta_{a b}^{(1)}\left(Z_{1} \Theta_{a b}^{(1)}-2 \varepsilon K_{a b}\right) \\
& \left.-Z_{3} \Theta_{a b}^{(3)}\left(Z_{3} \Theta_{a b}^{(3)}-2 \varepsilon K_{a b}\right)\right] .
\end{aligned}
$$

When combined with (2.10) one gets:

$$
\begin{aligned}
\hat{\nabla}^{2} Z_{I}= & -\frac{1}{4} Z_{J}^{-1} Z_{K}^{-1}\left[Z_{J} \Theta_{a b}^{(J)}\left(Z_{J} \Theta_{a b}^{(J)}-2 \varepsilon K_{a b}\right)\right. \\
& \left.+Z_{K} \Theta_{a b}^{(K)}\left(Z_{K} \Theta_{a b}^{(K)}-2 \varepsilon K_{a b}\right)-Z_{I} \Theta_{a b}^{(I)}\left(Z_{I} \Theta_{a b}^{(I)}-2 \varepsilon K_{a b}\right)\right],
\end{aligned}
$$

where $\{I, J, K\}=\{1,2,3\}$ are all distinct and these indices are not summed. 


\subsection{Maxwell equations}

To give the Maxwell equations it is convenient to define:

$$
\mathcal{R}_{ \pm}^{(I)} \equiv \frac{1}{2} \varepsilon Z_{I}\left(\Theta^{(I)} \pm \varepsilon *_{4} \Theta^{(I)}\right), \quad \mathcal{P}_{ \pm} \equiv \frac{1}{2}\left(K \pm \varepsilon *_{4} K\right)-\frac{1}{2} \sum_{M=1}^{3} \mathcal{R}_{ \pm}^{(M)}
$$

with no sum on $I$. Note that $\mathcal{P}=\mathcal{P}_{+}+\mathcal{P}_{-}$. The parameter, $\varepsilon$, satisfies $\varepsilon^{2}=1$ and so determines whether these combinations are self-dual or anti-self-dual.

The Maxwell equations are:

$$
d *_{5}\left(Q_{I J} F^{J}\right)=\frac{1}{4} C_{I J K} F^{J} \wedge F^{K},
$$

with $F^{I}=d A^{I}$. Using the Ansatz (2.7) one obtains two types of terms: (i) a four form on the four-dimensional base and (ii) $e^{0}$ wedged into a three form on the four-dimensional base. The former generates the following equations for $\hat{\nabla}^{2} Z_{I}$ :

$$
\hat{\nabla}^{2} Z_{I}=\varepsilon *_{4}\left[\Theta^{(J)} \wedge \Theta^{(K)}+Z^{-3} Z_{I} K \wedge\left(K+\varepsilon *_{4} K+2 \mathcal{R}_{-}^{(I)}-\varepsilon \sum_{M=1}^{3} Z_{M} \Theta^{(M)}\right)\right] .
$$

Combining this with (2.19) one obtains three algebraic constraints on the forms $\mathcal{P}_{+}$ and $\mathcal{R}_{ \pm}^{(M)}$ :

$$
\mathcal{P}_{+} \wedge \mathcal{P}_{+}+\mathcal{P}_{+} \wedge \mathcal{R}_{+}^{(I)}+\frac{1}{4}\left(\mathcal{R}_{-}^{(I)}-\mathcal{R}_{-}^{(J)}+\mathcal{R}_{-}^{(K)}\right) \wedge\left(\mathcal{R}_{-}^{(I)}+\mathcal{R}_{-}^{(J)}-\mathcal{R}_{-}^{(K)}\right)=0,
$$

where, once again, $\{I, J, K\}=\{1,2,3\}$ are all distinct and these indices are not summed.

The second set of Maxwell equations can be written as:

$$
d\left(Z^{-3} Z_{I}\left(K+\varepsilon *_{4} K+2 \mathcal{R}_{-}^{(I)}-\varepsilon \sum_{M=1}^{3} Z_{M} \Theta^{(M)}\right)\right)=0,
$$

where the index $I$ isn't summed.

\section{Solving the system}

\subsection{General results}

Using the equations of motion one can easily show that:

$$
d\left(\left(Z_{1} Z_{2} Z_{3}\right)^{-1} \mathcal{P}_{+}\right)=0,
$$

and hence one may write

$$
\mathcal{P}_{+}=\left(Z_{1} Z_{2} Z_{3}\right) \omega_{+}^{(0)}
$$

where $\omega_{+}^{(0)}$ is harmonic.

One can simplify some of the Maxwell equations by introducing some additional forms, $\omega_{-}^{(I)}$ defined by:

$$
\frac{1}{2} \varepsilon\left(\Theta^{(I)}-\varepsilon *_{4} \Theta^{(I)}\right) \equiv C_{I J K} Z_{J} \omega_{-}^{(K)} .
$$


Since $Z_{1} Z_{2} Z_{3} \neq 0$, this transformation is invertible and so we have made no additional assumptions. In terms of these new $\varepsilon$-anti-self-dual forms, the Maxwell equations (2.24) become:

$$
d *_{4} \omega_{-}^{(I)}=\left(Z_{1} Z_{2} Z_{3}\right)^{-1} d Z_{I} \wedge \mathcal{P}_{+}
$$

and (2.23) becomes:

$$
\mathcal{P}_{+} \wedge \mathcal{P}_{+}+\mathcal{P}_{+} \wedge \mathcal{R}_{+}^{(I)}+\left(Z_{1} Z_{2} Z_{3}\right) Z_{I} \omega_{-}^{(J)} \wedge \omega_{-}^{(K)}=0
$$

where $\{I, J, K\}=\{1,2,3\}$ are all distinct and these indices are not summed.

To simplify Einstein's equations, introduce the function, $\mathcal{T}_{a b}$, of a pair of two forms that is defined by:

$$
\mathcal{T}_{a b}(X, Y) \equiv \frac{1}{2}\left(X_{a c} Y_{b c}+X_{b c} Y_{a c}\right)-\frac{1}{4} \delta_{a b} X_{c d} Y_{c d}
$$

In particular, $\mathcal{T}_{a b}(F, F)$ is the energy momentum tensor associated with the Maxwell field, $F$. Note that if $X_{ \pm}$and $Y_{ \pm}$are the self-dual and anti-self dual parts of $X$ and $Y$, then

$$
\mathcal{T}_{a b}\left(X_{ \pm}, Y_{ \pm}\right)=0, \quad \mathcal{T}_{a b}(X, Y)=\mathcal{T}_{a b}\left(X_{+}, Y_{-}\right)+\mathcal{T}_{a b}\left(X_{-}, Y_{+}\right) .
$$

Using this in the Einstein equations (2.14), one obtains:

$$
\hat{R}_{a b}=2 Z^{-3} \mathcal{T}_{a b}\left(\mathcal{P}_{+}, \mathcal{P}_{-}\right)-\sum_{I=1}^{3} \mathcal{T}_{a b}\left(\frac{1}{2} \varepsilon\left(\Theta^{(I)}+\varepsilon *_{4} \Theta^{(I)}\right), \omega_{-}^{(I)}\right) .
$$

Thus far we have made no assumptions other than our floating brane Ansatz.

\subsection{A simple assumption}

The equations of motion dramatically simplify if one takes:

$$
\mathcal{P}_{+} \equiv 0,
$$

which is, of course, consistent with (3.1) and thus with the equations of motion. We will henceforth assume that (3.9) is true.

One then finds from (3.4) and (3.5) that the forms $\omega_{-}^{(I)}$ must be harmonic and satisfy

$$
\omega_{-}^{(I)} \wedge \omega_{-}^{(J)}=0, \quad I \neq J
$$

There are two obvious ways to satisfy this condition:

- (i) Take $\omega_{-}^{(1)}=\omega_{-}^{(2)}=0$ and $\omega_{-}^{(3)}$ to be an arbitrary $\varepsilon$-anti-self-dual harmonic form.

- (ii) Take the manifold to be hyper-Kähler and let each of the $\omega_{-}^{(I)}$ be a constant multiple of one the three harmonic two forms associated with the three complex structures. ${ }^{6}$

Continuing with the implications of (3.9), one finds that the equations for the scalars (2.22) reduce to:

$$
\hat{\nabla}^{2} Z_{I}=\varepsilon *_{4}\left[\Theta^{(J)} \wedge \Theta^{(K)}-\omega_{-}^{(I)} \wedge\left(K-\varepsilon *_{4} K\right)\right],
$$

\footnotetext{
${ }^{6}$ There might be an interesting generalization of (ii) to quaternionic-Kähler spaces.
} 
and Einstein's equations collapse to

$$
\hat{R}_{a b}=-\frac{1}{2} \varepsilon \sum_{I=1}^{3} \mathcal{T}_{a b}\left(\left(\Theta^{(I)}+\varepsilon *_{4} \Theta^{(I)}\right), \omega_{-}^{(I)}\right) .
$$

Note that the Ricci tensor depends only upon the four-dimensional electromagnetic fluxes.

\subsection{A further assumption and a linear system}

We now make a further assumption, that condition (i) above is satisfied: Hence $\omega_{-}^{(1)}=$ $\omega_{-}^{(2)}=0$ and $\omega_{-}^{(3)}$ is an arbitrary $\varepsilon$-anti-self-dual harmonic form. Then the equations become

$$
\begin{aligned}
& \left(\Theta^{(1)}-\varepsilon *_{4} \Theta^{(1)}\right)=2 \varepsilon Z_{2} \omega_{-}^{(3)}, \quad\left(\Theta^{(2)}-\varepsilon *_{4} \Theta^{(2)}\right)=2 \varepsilon Z_{1} \omega_{-}^{(3)}, \\
& \left(\Theta^{(3)}-\varepsilon *_{4} \Theta^{(3)}\right)=0 .
\end{aligned}
$$

Thus $\Theta^{(3)}$ is a harmonic, $\varepsilon$-self-dual two form.

The background geometry must be chosen so that

$$
\hat{R}_{a b}=-\varepsilon \mathcal{T}_{a b}\left(\Theta^{(3)}, \omega_{-}^{(3)}\right) \equiv \frac{1}{2}\left(\mathcal{F}_{a c} \mathcal{F}_{b c}-\frac{1}{4} \delta_{a b} \mathcal{F}_{c d} \mathcal{F}_{c d}\right)
$$

where $\mathcal{F}$ is defined by

$$
\mathcal{F} \equiv \Theta^{(3)}-\varepsilon \omega_{-}^{(3)} .
$$

Note that this Maxwell field must be harmonic.

To find a full solution of the supergravity equations of motion one must start from a Euclidean "electrovac" solution to U(1) Einstein-Maxwell theory. The metric of this solution will be the base metric of the full geometry, and the self- and anti-self-dual parts of the electrovac Maxwell field determine $\Theta^{(3)}$ and $\omega_{-}^{(3)}$. Note that both these forms must be closed, as a consequence of the Maxwell equations and Bianchi identities for $\mathcal{F}$. They will therefore automatically satisfy equations (3.13) and (3.4) under assumption (i). Conversely, given any solution to our equations, one can always repackage $\Theta^{(3)}$ and $\omega_{-}^{(3)}$ into a Maxwell field that satisfies (3.14), and obtain an electrovac solution.

Given $\Theta^{(3)}$ and $\omega_{-}^{(3)}$, we then need to solve the following pairs of equations:

$$
\begin{array}{rlrl}
\hat{\nabla}^{2} Z_{1} & =\varepsilon *_{4}\left[\Theta^{(2)} \wedge \Theta^{(3)}\right], & & \left(\Theta^{(2)}-\varepsilon *_{4} \Theta^{(2)}\right)=2 \varepsilon Z_{1} \omega_{-}^{(3)} ; \\
\hat{\nabla}^{2} Z_{2}=\varepsilon *_{4}\left[\Theta^{(1)} \wedge \Theta^{(3)}\right], & & \left(\Theta^{(1)}-\varepsilon *_{4} \Theta^{(1)}\right)=2 \varepsilon Z_{2} \omega_{-}^{(3)} .
\end{array}
$$

Since $\Theta^{(3)}$ and $\omega_{-}^{(3)}$ are already known, (3.16) represents a coupled linear system for $\Theta^{(2)}$ and $Z_{1}$ and (3.17) represents a coupled linear system for $\Theta^{(1)}$ and $Z_{2}$. In solving these systems one should, of course, remember that the $\Theta^{(I)}$ should also satisfy the (linear) Bianchi identities $d \Theta^{(1)}=0$.

Once one knows the solutions of the equations above, one must solve the equations for $Z_{3}$ and $K=d k$, which, amazingly enough, are also linear:

$$
\begin{aligned}
K+\varepsilon *_{4} K & =\frac{1}{2} \varepsilon \sum_{I} Z_{I}\left(\Theta^{(I)}+\varepsilon *_{4} \Theta^{(I)}\right), \\
\hat{\nabla}^{2} Z_{3} & =\varepsilon *_{4}\left[\Theta^{(1)} \wedge \Theta^{(2)}-\omega_{-}^{(3)} \wedge\left(K-\varepsilon *_{4} K\right)\right] .
\end{aligned}
$$


Hence, starting from an Euclidean electrovac solution one can build a full solution of fivedimensional $\mathrm{U}(1)^{3}$ ungauged supergravity by following a linear procedure, much like one does for BPS and almost-BPS solutions. Note however that our class of solutions is much larger, and includes the BPS and almost-BPS solutions. The latter merge by restricting the electric fields of the Euclidean electrovac solution to be self- or anti-self-dual, and thus the base space becomes Ricci-flat. We now explain in detail how this happens.

If $\omega_{-}^{(3)}=0$, then one has

$$
\Theta^{(I)}=\varepsilon *_{4} \Theta^{(I)}, \quad \hat{\nabla}^{2} Z_{I}=\varepsilon \frac{1}{2} C_{I J K} *_{4} \Theta^{(J)} \wedge \Theta^{(K)}, \quad \mathcal{P}_{+}=0 .
$$

For $\varepsilon=1$, these are just the BPS equations of [18, 19]. For $\varepsilon=-1$ they become the almost-BPS equations of [20]. Nevertheless, note that (3.12) implies that the base space of these solutions does not need to be hyper-Kähler, but only Ricci flat:

$$
\hat{R}_{a b}=0 .
$$

Hence, both the BPS and the almost-BPS equations allow for non-trivial non-BPS solutions with non-hyper-Kähler bases. Examples of such solutions are constructed in [23]. The fact that the base only needs to be Ricci-flat is not so strange: The hyper-Kähler condition originally arose because one wanted to preserve supersymmetry, however, Einstein's equations, (3.8), only care about the Ricci tensor of the base. It is also interesting to note that there also exist solutions with Ricci-flat bases that have $\omega_{-}^{(3)} \neq 0$ and $\Theta^{(3)}=0$. These solutions can in principle be obtained by recycling the known BPS and almost-BPS solutions, and their physics is worth exploring further.

\section{Israel-Wilson metrics}

We now examine, in more detail, the linear system of equations found in section 3.3, and solve it for a special class of electrovac base spaces that have a translational U(1) isometry: the Israel-Wilson spaces. From a four-dimensional perspective, the highly nontrivial particular solution we find describes a non-BPS two-centered solution where one of the centers is a locally-BPS D6-D4-D2-D0 black hole and the other center is a $\overline{\mathrm{D} 6}$ brane.

\subsection{The Israel-Wilson background}

The starting ingredient for constructing non-trivial solutions using the procedure outlined above is an Euclidean electrovac solution that satisfies (3.14) and that has a non-trivial harmonic form, $\omega_{-}^{(3)}$. An interesting choice for such a background is an Israel-Wilson (IW) metric [51-54]:

$$
d s_{4}^{2}=\left(V_{+} V_{-}\right)^{-1}(d \psi+\vec{A} \cdot d \vec{y})^{2}+\left(V_{+} V_{-}\right)\left(d y_{1}^{2}+d y_{2}^{2}+d y_{3}^{2}\right),
$$

where

$$
\vec{\nabla} \times \vec{A}=V_{-} \vec{\nabla} V_{+}-V_{+} \vec{\nabla} V_{-},
$$


and the functions $V_{ \pm}$are required to be harmonic on the $\mathbb{R}^{3}$ base. Introducing the frames:

$$
\hat{e}^{1}=\left(V_{+} V_{-}\right)^{-\frac{1}{2}}(d \psi+\vec{A} \cdot d \vec{y}), \quad \hat{e}^{a+1}=\left(V_{+} V_{-}\right)^{\frac{1}{2}} d y^{a}, \quad a=1,2,3,
$$

the background Maxwell field is given by [54]:

$$
\begin{aligned}
\mathcal{F} & \equiv \frac{1}{2} \mathcal{F}_{a b} \hat{e}^{a} \wedge \hat{e}^{b} \\
& =\left[\partial_{a}\left(V_{+}^{-1}-V_{-}^{-1}\right)\right] e^{1} \wedge \hat{e}^{a+1}+\frac{1}{2} \epsilon_{a b c}\left[\partial_{a}\left(V_{+}^{-1}+V_{-}^{-1}\right)\right] e^{b+1} \wedge \hat{e}^{c+1}
\end{aligned}
$$

This background then satisfies equation (3.14).

\subsection{Harmonic forms}

Define the sets of two-forms:

$$
\Omega_{ \pm}^{(a)} \equiv \hat{e}^{1} \wedge \hat{e}^{a+1} \pm \frac{1}{2} \epsilon_{a b c} \hat{e}^{b+1} \wedge \hat{e}^{c+1}, \quad a=1,2,3 .
$$

The Maxwell field of the Israel-Wilson solution is then:

$$
\mathcal{F}=\left(\partial_{a}\left(V_{+}^{-1}\right)\right) \Omega_{+}^{(a)}-\left(\partial_{a}\left(V_{-}^{-1}\right)\right) \Omega_{-}^{(a)},
$$

from which one can read off (up to an irrelevant, overall sign) the harmonic forms, $\Theta^{(3)}$ and $\omega_{-}^{(3)}$, using (3.15). However, it is easy to see that (4.4) is not the most general Maxwell field one can have for this base. Introducing two arbitrary harmonic functions $K_{ \pm}$on $\mathbb{R}^{3}$, the two-forms:

$$
\Theta_{ \pm} \equiv-\sum_{a=1}^{3}\left(\partial_{a}\left(V_{ \pm}^{-1} K_{ \pm}\right)\right) \Omega_{ \pm}^{(a)}
$$

are also harmonic and self-dual, or anti-self-dual respectively. These forms have (local) potentials:

$$
a_{ \pm}=\frac{K_{ \pm}}{V_{ \pm}}(d \psi+\vec{A} \cdot d \vec{y})+\vec{b}_{ \pm} \cdot d \vec{y}, \quad \vec{\nabla} \times \vec{b}_{ \pm}= \pm\left(K_{ \pm} \vec{\nabla} V_{\mp}-V_{\mp} \vec{\nabla} K_{ \pm}\right) .
$$

From now on we choose $\varepsilon=1$. The equations for $(\varepsilon=-1)$ can be simply obtained by exchanging $V_{+}$and $V_{-}$. The two-form, $\Theta^{(3)}$, is then self-dual while $\omega_{-}^{(3)}$ is anti-self-dual.

One can try to obtain a more general solution for $\Theta^{(3)}$ and $\omega_{-}^{(3)}$ by taking:

$$
\Theta^{(3)}=d\left(\frac{K_{+}}{V_{+}}(d \psi+\vec{A})+\vec{b}_{+}\right), \quad \omega_{-}^{(3)}=d\left(\frac{K_{-}}{V_{-}}(d \psi+\vec{A})+\vec{b}_{-}\right) .
$$

The Einstein-Maxwell electrovac equations (3.14) are then solved if, and only if

$$
\partial_{i}\left(\frac{K_{+}}{V_{+}}\right) \partial_{j}\left(\frac{K_{-}}{V_{-}}\right)=\left(\partial_{i} V_{+}^{-1}\right)\left(\partial_{j} V_{-}^{-1}\right)
$$

for $i, j=1,2,3$. Hence, one can apparently obtain a more general electrovac Israel-Wilson base by using the solutions to this equation:

$$
K_{-}=\beta V_{-}-\alpha, \quad K_{+}=\delta V_{+}-\gamma
$$


with $\alpha, \beta, \gamma, \delta$ constants satisfying the constraint $\alpha \gamma=1$. However, one can easily see that $\beta$ and $\delta$ are "pure gauge" constants, since they make no contribution to the Maxwell fields (4.7). We therefore set $\beta=0$, which implies that $K_{-}$is constant. We could, of course, do the same with $K_{+}$, however, we will find it useful in the next sub-section to keep $\delta \neq 0$.

We should also note that the foregoing discussion no longer applies if either $V_{-}$or $V_{+}$ are constant, because the solutions to (4.10) are then different from those in (4.11). We will partially address this situation later in the paper, and we leave a more general analysis for further investigation. Given that $K_{-}=-\alpha$, the two-form $\omega_{-}^{(3)}$ is a constant multiple of the natural anti-self-dual two-form on the Israel-Wilson base space, $\left(\partial_{a}\left(V_{-}^{-1}\right)\right) \Omega_{-}^{(a)}$.

\subsection{The linear system}

We now solve the linear system for the other fields. We write $\Theta^{(1)}$ and $\Theta^{(2)}$ in the form:

$$
\Theta^{(1)}=d\left(\frac{K_{1}}{V_{+}}(d \psi+A)+b_{1}\right), \quad \Theta^{(2)}=d\left(\frac{K_{2}}{V_{+}}(d \psi+A)+b_{2}\right)
$$

where $K_{1}, K_{2}, b_{1}$ and $b_{2}$ are unknown functions and one-forms on the $\mathbb{R}^{3}$ base and determine the dipole charges of the solution. Writing the equations (3.16) in the IW base, we obtain

$$
\begin{aligned}
\nabla^{2} K_{2} & =\frac{2 \alpha}{V_{-}} \vec{\nabla} \cdot\left(\frac{V_{+}}{V_{-}} Z_{1} \vec{\nabla} V_{-}\right) \\
\nabla^{2} Z_{1} & =V_{-} \nabla^{2}\left(\frac{K_{2} K_{+}}{V_{+}}\right)-2 \alpha \vec{\nabla} \cdot\left(\frac{Z_{1} K_{+}}{V_{-}} \vec{\nabla} V_{-}\right)
\end{aligned}
$$

with $b_{2}$ given by

$$
\vec{\nabla} \times \vec{b}_{2}=-V_{-} \vec{\nabla} K_{2}+K_{2} \vec{\nabla} V_{-}+2 \alpha \frac{V_{+}}{V_{-}} Z_{1} \vec{\nabla} V_{-}
$$

The corresponding system for $Z_{2}$ and $\Theta^{(1)}$ is:

$$
\begin{aligned}
\nabla^{2} K_{1} & =\frac{2 \alpha}{V_{-}} \vec{\nabla} \cdot\left(\frac{V_{+}}{V_{-}} Z_{2} \vec{\nabla} V_{-}\right) \\
\nabla^{2} Z_{2} & =V_{-} \nabla^{2}\left(\frac{K_{1} K_{+}}{V_{+}}\right)-2 \alpha \vec{\nabla} \cdot\left(\frac{Z_{2} K_{+}}{V_{-}} \vec{\nabla} V_{-}\right) \\
\vec{\nabla} \times \vec{b}_{1} & =-V_{-} \vec{\nabla} K_{1}+K_{1} \vec{\nabla} V_{-}+2 \alpha \frac{V_{+}}{V_{-}} Z_{2} \vec{\nabla} V_{-}
\end{aligned}
$$

We also need the equation for the last warp factor $Z_{3}$ and the angular momentum $k$. We decompose $k$ as usual:

$$
k=\mu(d \psi+A)+\omega
$$


Equations (3.18) and (3.19) for $\mu$ and $Z_{3}$ then give

$$
\begin{aligned}
\nabla^{2} Z_{3}= & V_{-} \nabla^{2}\left(\frac{K_{1} K_{2}}{V_{+}}\right)-2 \alpha \vec{\nabla} \cdot\left(\frac{Z_{1} K_{1}+Z_{2} K_{2}}{V_{-}} \vec{\nabla} V_{-}\right) \\
& +4 \alpha \frac{V_{+}}{V_{-}} \vec{\nabla} \cdot\left(\mu \vec{\nabla} V_{-}\right)-2 \alpha \frac{V_{+} Z_{I}}{V_{-}} \vec{\nabla} \cdot\left(\frac{K_{I}}{V_{+}} \vec{\nabla} V_{-}\right) \\
& +2 \alpha^{2} V_{+} Z_{1} Z_{2} \nabla^{2}\left(\frac{1}{V_{-}}\right), \\
\nabla^{2}\left(V_{-} \mu\right)= & \frac{1}{V_{+}} \vec{\nabla} \cdot\left(V_{-} V_{+} Z_{I} \vec{\nabla}\left(\frac{K_{I}}{V_{+}}\right)\right)-\frac{2 \alpha}{V_{+}} \vec{\nabla} \cdot\left(\frac{V_{+} Z_{1} Z_{2}}{V_{-}} \vec{\nabla} V_{-}\right)
\end{aligned}
$$

where $\omega$ is given by:

$$
\vec{\nabla} \times \vec{\omega}=V_{+}^{2} \vec{\nabla}\left(\frac{V_{-}}{V_{+}} \mu\right)-V_{+} V_{-} Z_{I} \vec{\nabla}\left(\frac{K_{I}}{V_{+}}\right)+2 \alpha \frac{V_{+} Z_{1} Z_{2}}{V_{-}} \vec{\nabla} V_{-} .
$$

As usual, (4.21) is the integrability equation for (4.22).

\subsection{An explicit example: a non-BPS black hole in an Israel-Wilson metric}

We now have all the tools to find explicit solutions with an Israel-Wilson base space. Here we will present an M-theory solution that corresponds, in type IIA string theory, to a $\mathrm{D} 6 \mathrm{D} 4^{3} \mathrm{D} 2^{3} \mathrm{D} 0$ black hole in a $\overline{\mathrm{D} 6}$ background. We parameterize the flat, three-dimensional $\mathbb{R}^{3}$ base space using spherical coordinates $(r, \theta, \phi)$ and put the $\overline{\mathrm{D} 6}$ brane at the origin of the space and the black hole at a distance $R$ from the origin. We denote polar coordinates centered at the black hole position by $\left(\Sigma, \theta_{\Sigma}\right)$. Their relation to the polar coordinates $(r, \theta)$ centered at the origin is:

$$
\Sigma=\sqrt{r^{2}+R^{2}-2 r R \cos \theta}, \quad \cos \theta_{\Sigma}=\frac{r \cos \theta-R}{\Sigma} .
$$

For $V_{+}=1$, we want the space to be Taub-NUT, and thus we take $V_{-}$to be

$$
V_{-}=1+\frac{Q_{\overline{6}}}{r} .
$$

The parameter, $Q_{\overline{6}}$, is $\overline{\mathrm{D} 6}$ or the $\overline{\mathrm{KK}}$-monopole charge of the space. ${ }^{7}$ The function, $K_{+}$, is harmonic and corresponds to one of the M5 charges of the solution:

$$
K_{+}=K_{3}=\frac{d_{3}}{\Sigma}
$$

For convenience, we will change notation throughout the rest of the paper, and refer to $K_{-}$as $K_{3}$. The relation (4.11) then forces $V_{+}$to have a pole at the black hole location. Assuming the space to be asymptotically flat (asymptotic to $\mathbb{R}^{3} \times S^{1}$ ) means that the constant in $V_{+}$to be finite, and we set it to 1 for convenience. Hence,

$$
V_{+}=1+\alpha K_{+}=1+\frac{\alpha d_{3}}{\Sigma} \equiv 1+\frac{Q_{6}}{\Sigma},
$$

\footnotetext{
${ }^{7}$ We will explain below why we refer to this as $\overline{\mathrm{D} 6}$ and not as D6 charge.
} 
where $\alpha$ was introduced in (4.11), and we have defined $Q_{6} \equiv \alpha d_{3}$. Thus, the black hole has a finite D6 (or $\mathrm{KKm}$ ) charge. The associated vector fields are

$$
\begin{aligned}
A & =Q_{6} \frac{r \cos \theta-R}{\Sigma} d \phi+Q_{6} Q_{\overline{6}} \frac{r-R \cos \theta}{\Sigma} d \phi-Q_{\overline{6}} \cos \theta d \phi, \\
b_{3} & =-d_{3} \frac{r \cos \theta-R}{\Sigma} d \phi-Q_{\overline{6}} d_{3} \frac{r-R \cos \theta}{\Sigma} d \phi
\end{aligned}
$$

The system (4.13) and (4.14) is not completely straightforward to solve, but, as explained in the previous section, it is linear in the unknowns $K_{2}$ and $Z_{1}$. We find the following solution:

$$
\begin{aligned}
Z_{1} & =\frac{1}{V_{+}}\left(1+\frac{Q_{1}}{\Sigma}+\frac{d_{2} d_{3}}{\Sigma^{2}}\left(1+\frac{Q_{\overline{6}} r}{R^{2}}\right)\right), \\
K_{2} & =V_{+}\left(\frac{d_{2}}{\Sigma}-\alpha \frac{Z_{1}}{V_{-}}\right),
\end{aligned}
$$

and similarly for $Z_{2}$ and $K_{1}$. Here we have also introduced the dipole charge $d_{2}$ associated to $K_{2}$, and the electric charge $Q_{1}$ of the hole, associated to $Z_{1}$. The vector field $b_{2}$ is then given by

$$
b_{2}=-\left(d_{2}-\alpha Q_{1}\right) \frac{r \cos \theta-R}{\Sigma} d \phi-Q_{\overline{6}} d_{2} \frac{r-R \cos \theta}{\Sigma} d \phi+d_{2} Q_{6} Q_{\overline{6}} \frac{\cos \theta}{\Sigma^{2}} d \phi .
$$

The solution to the last system of equations, (4.20) and (4.21), is:

$$
\begin{aligned}
\mu= & \frac{1}{V_{+} V_{-}}\left(\frac{m}{\Sigma}+\frac{\tilde{m}}{r}+\frac{V_{-}\left(d_{1}+d_{2}+d_{3}\right)}{2 \Sigma}+\frac{Q_{I} d_{I}}{2 \Sigma^{2}}+Q_{\overline{6}} Q_{I} d_{I} \frac{\cos \theta}{2 R \Sigma^{2}}\right. \\
& \left.+\frac{C_{I J K}}{6} d_{I} d_{J} d_{K}\left[\left(1+\frac{Q_{\overline{6}}^{2}}{R^{2}}\right)\left(\frac{r \cos \theta}{R \Sigma^{3}}+\lambda \frac{r \cos \theta-R}{R \Sigma^{3}}\right)+Q_{\overline{6}} \frac{3 r^{2}+R^{2}}{2 R^{2} r \Sigma^{3}}\right]\right)-\alpha \frac{Z_{1} Z_{2}}{V_{-}}, \\
Z_{3}= & V_{+}\left(1+\frac{Q_{3}}{\Sigma}+\frac{d_{1} d_{2}}{\Sigma^{2}}\left(1+\frac{Q_{\overline{6}} r}{R^{2}}\right)\right)-2 \alpha V_{+} \mu-\alpha^{2} \frac{V_{+} Z_{1} Z_{2}}{V_{-}}
\end{aligned}
$$

and

$$
\begin{aligned}
\omega= & -\left[\kappa-m \frac{r \cos \theta-R}{\Sigma}-\tilde{m} \cos \theta+\frac{d_{1}+d_{2}+d_{3}}{2} \frac{r \cos \theta-R}{\Sigma}+Q_{\overline{6}} \frac{d_{1}+d_{2}+d_{3}}{2} \frac{r-R \cos \theta}{R \Sigma}\right. \\
& +Q_{\overline{6}} Q_{I} d_{I} \frac{r \sin ^{2} \theta}{2 R \Sigma^{2}}+\left(1+\frac{Q_{\overline{6}}^{2}}{R^{2}}\right) \frac{C_{I J K}}{6} d_{I} d_{J} d_{K}(1+\lambda) \frac{r^{2} \sin ^{2} \theta}{R \Sigma^{3}} \\
& \left.+Q_{\overline{6}} \frac{C_{I J K}}{6} d_{I} d_{J} d_{K} \frac{r\left(3 R^{2}+r^{2}\right)-R\left(3 r^{2}+R^{2}\right) \cos \theta}{2 R^{3} \Sigma^{3}}\right] d \phi .
\end{aligned}
$$

The constants $m, \tilde{m}, \kappa$ and $\lambda$ represent homogeneous solutions that are fixed by regularity:

$$
\begin{aligned}
& m=\left(1+\frac{Q_{\overline{6}}}{R}\right) \frac{d_{1}+d_{2}+d_{3}}{2}+\frac{C_{I J K}}{6} \frac{Q_{\overline{6}} d_{I} d_{J} d_{K}}{2 R^{3}}, \\
& \tilde{m}=\kappa=-Q_{\overline{6}}\left(\frac{d_{1}+d_{2}+d_{3}}{2 R}+\frac{C_{I J K}}{6} \frac{d_{I} d_{J} d_{K}}{2 R^{3}}\right) . \\
& \lambda=-\frac{R^{2}}{R^{2}+Q_{\overline{6}}^{2}} .
\end{aligned}
$$

The reason for this regularity conditions will become clear in the section 5.2. 


\subsection{The BPS and almost-BPS limits of solutions with an Israel-Wilson base}

The solution presented here seems to be somewhat complicated, but its physical interpretation is rather straightforward. Take first $V_{+}$to be 1 (by setting the parameter $\alpha$ to zero). As we already remarked, the metric then becomes the usual Taub-NUT metric, with negative orientation. Looking at the complete solution, we see that it becomes the non-BPS black ring in Taub-NUT solution found in [21]. ${ }^{8}$ From a four-dimensional perspective this is a two-center solution where one center is a D4-D2-D0 black hole located at $z=R$ and the other is a pure $\overline{\mathrm{D} 6}$ brane located at $r=0$. Despite the fact that both objects are locally$\mathrm{BPS}$, the relative orientation of the $\overline{\mathrm{D} 6}$ and the three D2 branes (which determine, locally, the Killing spinors of the D4-D2-D0 black hole) makes the full configuration non-BPS.

On the other hand, setting $Q_{\overline{6}}$ to zero, and hence $V_{-}=1$, one can see that the solution becomes BPS and describes a four-dimensional black hole with D6, D4, D2 and D0 charges. The singular part in the D4 harmonic function can be traded, via a gauge transformation $[27,57]$ for a non-trivial Wilson line at infinity, and thus this black hole is in fact a BMPV black hole located at the tip of Taub-NUT (which is now located at at $z=R$ because we set $Q_{\overline{6}}=0$ ), or a single-center D6-D2-D0 black hole from a four-dimensional perspective. For this solution the relative orientation of the D6 and D2 branes match, and the solution preserves four supercharges. These two limits are summarized in the figure 1 . If one now takes both the D6 and the $\overline{\mathrm{D} 6}$ charges to zero, the solution becomes a BPS D4D2-D0 four-dimensional black hole, which lifts to a BPS three-charge three-dipole-charge black string in $\mathbb{R}^{3,1} \times S^{1}[31]$.

Having taken these limits, it is now clear that the general solution with an IsraelWilson base describes a two-center configuration, where one of the centers has D6,D4,D2, and D0 charges and is locally-BPS, and the other has $\overline{\mathrm{D} 6}$ charge. Of course, an IsraelWilson solution with multiple D6 branes of opposite orientations is only possible when other charges and fluxes are turned on (3.14). Indeed, the D6 and $\overline{\mathrm{D} 6}$ charges attract each other and in the absence of other branes, there is nothing to balance this attraction. Introducing D4, D2 and D0 branes creates new interactions: the D4 branes are also attracted, the D2's feel no force, and the D0's are repelled, and thus balance becomes possible.

Note that upon flipping the sign of $\varepsilon$ one could also obtain a solution where the D6 charge becomes anti-D6 charge and vice versa; this solution should describe an intrinsically non-BPS $\overline{\mathrm{D}} 6$-D2-D0 black hole in a background of a D6 brane that is mutually-BPS with respect to the three sets of $\mathrm{D} 2$ branes. When the $\overline{\mathrm{D} 6}$ charge is zero the solution should become a BPS black ring in Taub-NUT [32-34] and when the D6 charge is zero it should becomes the almost-BPS non-rotating $\overline{\mathrm{D} 6}$-D2-D0 black hole [28-30]. When both the $\overline{\mathrm{D} 6}$ and the D6 charges are zero, this solution should reduce again to the D4-D2-D0 BPS black hole whose five-dimensional lift is the M5-M2-P black string (or the infinite black ring) of [31].

\section{Spectral flow and the Israel-Wilson metric}

In this section we study the action of a spectral flow transformation [27] on the solution describing an almost-BPS black ring in Taub-NUT [21], and show that it yields the $\varepsilon=1$ solution with the Israel-Wilson base found in the previous section. We also argue that all

\footnotetext{
${ }^{8}$ In [21], the solution is written in the $\varepsilon=-1$ convention.
} 


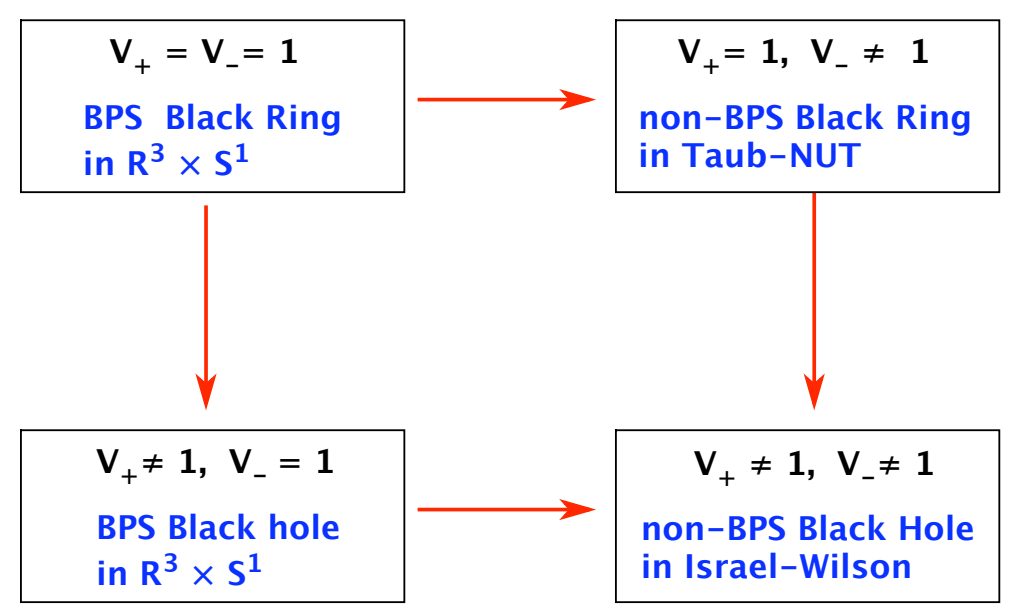

Figure 1. This diagram represents four classes of solutions that can be obtained from our solution for various values of the Israel-Wilson harmonic functions. When both $V_{+}$and $V_{-}$are constant, the solution describes a BPS black string in $\mathbb{R}^{3} \times S^{1}$. Turning on a $\overline{K K m}$ charge at the center of the space $\left(V_{-} \neq 1\right)$ the space becomes Taub-NUT, the black ring is non-BPS and the solution belongs to the almost-BPS Ansatz. Turning on a $K K m$ charge at the location of the ring, we obtain a BPS D6-D4-D2-D0 black hole. Turning on both types of $\mathrm{KKm}$ charges $\left(V_{+} \neq 1, V_{-} \neq 1\right)$, we obtain the more general non-BPS solution constructed here: a D6-D4-D2-D0 four-charge black hole in a $\overline{\mathrm{D} 6}$ background.

the solutions that are constructed starting from a Euclidean electrovac solution given by the Israel-Wilson metric can be generated by the spectral flow of a more-standard "almostBPS" solution.

\subsection{The D1-D5-P duality frame}

The spectral flow transformation presented in [27] is a very useful tool for generating new asymptotically $\mathbb{R}^{3,1} \times S^{1}$ solutions of five-dimensional $\mathrm{U}(1)^{3}$ ungauged supergravity (or of the STU model in four dimensions) by starting from other such solutions. In asymptotically $A d S_{3} \times S^{3}$ spaces this transformation is the gravity counterpart of a symmetry of the dual CFT, and it is most naturally performed upon dualizing the solution to the D1-D5-P duality frame [35-40]. In this frame the solution, which is invariant along the four internal directions wrapped by the D5 branes, corresponds to a solution of six-dimensional ungauged supergravity [41, 42]. The spatial section of the metric can be written as a $T^{2}$ fibration over an $\mathbb{R}^{3}$ base, where the $T^{2}$ is made up by the fiber of the Taub-NUT base space and by the (internal) direction common to the D1 and the D5 branes. Spectral flows can then be recast as simply a subgroup of the group, $\mathrm{SL}(2, \mathbb{Z})$, of global diffeomorphisms on this $T^{2}$. Thus, from a six-dimensional point of view, spectral flow is just a change of coordinates, mixing two different U(1)'s. However, upon dualizing back to the duality frame where the charges correspond to three sets of M2 branes, the resulting solution, which is again a solution of $\mathrm{U}(1)^{3}$ supergravity, differs rather non-trivially from the original one. ${ }^{9}$

\footnotetext{
${ }^{9}$ Note that to go from a six-dimensional supergravity solution to the final solution of five-dimensional supergravity one does not KK reduce the six-dimensional solution; rather one trivially uplifts it to a solution of type IIB supergravity, performs three T-dualities, then uplifts the resulting solution to M-theory, and then reads off the new solution of five-dimensional ungauged supergravity.
} 
To perform a spectral flow we need to find the metric and RR gauge-field of the solution dualized to the D1-D5-P duality frame. This can be achieved starting from the eleven-dimensional supergravity solution, dimensionally-reducing along one of the torus directions, and then performing three T-dualities, as explained in detail in [55]. This yields

$$
\begin{aligned}
d s^{2} & =-\frac{1}{\sqrt{Z_{1} Z_{2} Z_{3}}}(d t+k)^{2}+\frac{Z_{3}}{\sqrt{Z_{1} Z_{2}}}\left(d y+A^{3}\right)^{2}+\sqrt{Z_{1} Z_{2}} d s_{4}^{2}+\sqrt{\frac{Z_{2}}{Z_{1}}} \sum_{a=1}^{4} d x_{a}^{2}, \\
C^{(2)} & =A^{1} \wedge\left(d y+A^{3}\right)+B^{(1)} \wedge \frac{d t+k}{Z_{3}}+\gamma_{2},
\end{aligned}
$$

where

$$
A^{I}=B^{(I)}-\frac{d t+k}{Z_{I}}
$$

and

$$
d \gamma_{2}=*_{4} d Z_{2}-B^{(1)} \wedge \Theta^{(3)} .
$$

For convenience we take $\varepsilon=1$; the result for the other sign is equally straightforward to obtain.

\subsection{The action of spectral flow}

We start from the solution of the "almost BPS" equation presented in [21], corresponding to a non-BPS black ring ${ }^{10}$ and thus we assume that the base metric $d s_{4}^{2}$ has GH form:

$$
d s_{4}^{2}=V_{-}^{-1}(d \psi+\vec{A} \cdot d \vec{y})^{2}+V_{-} d s_{3}^{2}, \quad \vec{\nabla} \times \vec{A}=-\vec{\nabla} V_{-} .
$$

The one-form potentials are:

$$
B^{(I)}=K_{I}(d \psi+A)+b_{I}, \quad k=\mu(d \psi+A)+\omega,
$$

where $I=1,2,3$, the $K_{I}$ are harmonic and the $b_{I}$ satisfy the equation:

$$
\vec{\nabla} \times \vec{b}_{I}=-V_{-} \vec{\nabla} K_{I}+K_{I} \vec{\nabla} V_{-} .
$$

In order to perform the spectral flow, we also need to decompose the two-form, $\gamma_{2}$, as

$$
\gamma_{2}=(d \psi+A) \wedge \gamma_{2}^{(\psi)}+\gamma_{2}^{(b)}
$$

where $\gamma_{2}^{(b)}$ is a two form on the three-dimensional space defined by $d s_{3}^{2}$.

Note that the equation for $Z_{2}$,

$$
d *_{4} d Z_{2}=\Theta^{(1)} \wedge \Theta^{(3)},
$$

implies

$$
\begin{aligned}
\nabla^{2} Z_{2} & =V_{-} \nabla^{2}\left(K_{1} K_{3}\right)=\vec{\nabla} \cdot\left(V_{-} \vec{\nabla}\left(K_{1} K_{3}\right)\right)-\vec{\nabla} V_{-} \cdot \vec{\nabla}\left(K_{1} K_{3}\right) \\
& =\vec{\nabla} \cdot\left(V_{-} \vec{\nabla}\left(K_{1} K_{3}\right)+\vec{A} \times \vec{\nabla}\left(K_{1} K_{3}\right)\right)
\end{aligned}
$$

\footnotetext{
${ }^{10}$ The solution in [21] was first written in the $\varepsilon=-1$ convention, ie $V=V_{+}$, and not the $\varepsilon=+1$ one we use here. One can rewrite the solution in the new convention by taking $V=V_{-}$, and changing the signs of the base-space vectors.
} 
and hence

$$
\vec{\nabla} Z_{2}=V_{-} \vec{\nabla}\left(K_{1} K_{3}\right)+\vec{A} \times \vec{\nabla}\left(K_{1} K_{3}\right)+\vec{\nabla} L_{2} .
$$

The equation satisfied by $\gamma_{2}$

$$
\vec{\nabla} \times \vec{\gamma}_{2}^{(\psi)}=\vec{\nabla} Z_{2}-V_{-} K_{1} \vec{\nabla} K_{3}+\vec{b}_{1} \times \vec{\nabla} K_{3}=\vec{\nabla} L_{2}-\vec{\nabla} \times\left(K_{3} \vec{b}_{1}+K_{1} K_{3} \vec{A}\right),
$$

implies

$$
\gamma_{2}^{(\psi)}=-K_{3} b_{1}-K_{1} K_{3} A+\hat{\gamma}_{2}^{(\psi)} \text { with } \vec{\nabla} \times \overrightarrow{\hat{\gamma}}_{2}^{(\psi)}=\vec{\nabla} L_{2} .
$$

Similarly one can define a two-form

$$
\gamma_{1}=(d \psi+A) \wedge \gamma_{1}^{(\psi)}+\gamma_{1}^{(b)}
$$

that satisfies

$$
d \gamma_{1}=*_{4} d Z_{1}-B^{(2)} \wedge \Theta^{(3)}
$$

One has

$$
\vec{\nabla} \times \vec{\gamma}_{1}^{(\psi)}=\vec{\nabla} Z_{1}-V_{-} K_{2} \vec{\nabla} K_{3}+b_{2} \times \vec{\nabla} K_{3}=\vec{\nabla} L_{1}-\vec{\nabla} \times\left(K_{3} \vec{b}_{2}+K_{2} K_{3} \vec{A}\right)
$$

which implies

$$
\gamma_{1}^{(\psi)}=-K_{3} b_{2}-K_{2} K_{3} A+\hat{\gamma}_{1}^{(\psi)} \text { with } \vec{\nabla} \times \overrightarrow{\hat{\gamma}}_{1}^{(\psi)}=\vec{\nabla} L_{1} .
$$

Spectral flow mixes the internal $\mathrm{U}(1)$ coordinate $y$, associated with the momentum charge, with the GH fiber, $\psi$. Explicitly, this is just the change of coordinates

$$
\psi \rightarrow \psi+\alpha y \text {. }
$$

To find the transformation of the metric coefficients, one performs the change of coordinates (5.18) and rewrites the metric and gauge field in the exact same form as (5.2). Defining the harmonic function $V_{+}$by

$$
V_{+}=1+\alpha K_{3},
$$

the transformed metric is

$$
d s_{4}^{2}=\left(V_{+} V_{-}\right)^{-1}(d \psi+\overrightarrow{\widetilde{A}} \cdot d \vec{y})^{2}+V_{+} V_{-} d s_{3}^{2}, \quad \widetilde{A}=A-\alpha b_{3} .
$$

Note that $\widetilde{A}$ now satisfies:

$$
\vec{\nabla} \times \overrightarrow{\widetilde{A}}=V_{-} \vec{\nabla} V_{+}-V_{+} \vec{\nabla} V_{-} .
$$

The rest of the fields can be recast in the exact same form as before, with the new coefficients (obtained after a fair amount of of algebra) given by:

$$
\begin{aligned}
& \widetilde{K}_{1}=K_{1}-\alpha \frac{Z_{2}}{V_{+} V_{-}}, \quad \widetilde{K}_{2}=K_{2}-\alpha \frac{Z_{1}}{V_{+} V_{-}}, \quad \widetilde{K}_{3}=\frac{K_{3}}{V_{+}}, \\
& \widetilde{b}_{1}=V_{+} b_{1}+\alpha \gamma_{2}^{(\psi)}, \quad \widetilde{b}_{2}=V_{+} b_{2}+\alpha \gamma_{1}^{(\psi)}, \quad \widetilde{b}_{3}=b_{3} \\
& \widetilde{Z}_{1}=\frac{Z_{1}}{V_{+}}, \quad \widetilde{Z}_{2}=\frac{Z_{2}}{V_{+}}, \quad \widetilde{Z}_{3}=V_{+} Z_{3}-2 \alpha \mu+\alpha^{2} \frac{Z_{1} Z_{2}}{V_{+} V_{-}} \\
& \widetilde{\mu}=\frac{1}{V_{+}}\left(\mu-\alpha \frac{Z_{1} Z_{2}}{V_{+} V_{-}}\right), \quad \widetilde{\omega}=\omega .
\end{aligned}
$$


This is exactly the solution with an Israel-Wilson base constructed in section 4 . In particular, the relation (5.19), which is the same as (4.26), between the harmonic function $V_{+}$corresponding to the D6 charge and one of the harmonic functions corresponding to D4 charge emerges directly from the spectral flow transformation.

While this approach to obtaining solutions is rather different from the one outlined in section 4 in that it does not involve starting from a non-trivial Einstein-Maxwell electrovac solution but from a Ricci-flat metric, the resulting solution is the same. This greatly simplifies the regularity analysis, as we know that spectral flow always transforms regular solutions into regular solutions. Hence the regularity of the D6-D4-D2-D0 black hole is ensured by the regularity of the non-BPS black ring in Taub-NUT, which yields the regularity conditions outlined in the previous section (4.35).

\subsection{Spectral flow and smooth horizonless multi-center solutions.}

One of the driving forces in our effort to construct large classes of multi-center non-BPS solutions is to obtain smooth horizonless solutions that have the same charges and mass as non-BPS black holes with a macroscopically-large horizon area. For BPS black holes, the existence of large classes of such solutions brings considerable support to the fact that these black holes should be thought of as statistical ensembles of horizonless configurations, thus realizing the fuzzball proposal (see [59-64] for reviews and some of the original works) for this class of black holes. We would like to extend this to non-BPS black holes.

The most obvious way to look for such non-BPS multi-center horizonless solutions is to use the almost-BPS Ansatz. However, in this Ansatz the anti-self-dual two-forms that one can turn on (for example the harmonic forms dual to the two-cycles of a multi-center Taub-NUT space) source strongly singular solutions to the equations of motion. Hence, at least at first glance, no smooth horizonless solutions exist.

The next obvious place to search for such solutions is in the floating-brane Ansatz. One obvious way to do this is to construct solutions explicitly when the base space is has an Israel-Wilson metric. For particular values of the D4, D2 and D0 charges, the D6 brane of the two-center floating-brane solution we constructed in section 4.4 can become a 16-supercharge fluxed D6 brane, and the five-dimensional lift of this D6- $\overline{\mathrm{D} 6}$ solution is completely smooth. The Israel-Wilson base space has one two-cycle running between the pole of $V_{+}$and the pole of $V_{-}$, and the non-trivial flux on this two-cycle is responsible for keeping the D6 and the $\overline{\mathrm{D} 6}$ apart, much like for BPS solutions.

Another way to obtain smooth horizonless solutions is to use spectral flow. It is well known that in the appropriate IIB frame a two-charge supertube with D1 and D5 charges corresponds to a completely regular geometry. Furthermore, using spectral flow, we can change coordinates and then dualize a BPS solution containing such a supertube in a multiTaub-NUT space into a completely regular multi-Taub-NUT five-dimensional solution with fluxes supported on bubbles [27]. On the other hand, a solution with multiple supertubes of different types (with different dipole charges) cannot simultaneously be dualized via one spectral flow to a smooth geometry. This needs to be done by three subsequent spectral flows, which transform every type of supertube into a Taub-NUT center. Since the neartube geometry is the same in a BPS and in an almost-BPS solution, we expect the spectral 
flow to transform multiple supertubes in an almost-BPS solution into a smooth non-BPS horizonless solution with multiple distinct fluxes supported on bubbles.

To illustrate this, consider a single supertube in a Taub-NUT geometry of "opposite orientation." That is, the base space is of the form (5.5) while the supertube magnetic dipoles are given by (5.6). If $K_{1}=K_{2}=0$ this supertube has only one dipole charge, and it can be arranged to give a completely regular geometry in six dimensions. However, as explained in [21], even if this solution is written as an almost-BPS solution, it still preserves four supersymmetries. ${ }^{11}$ One can now perform a spectral flow on this solution exactly as in section 5.2 and obtain a floating-brane solution with an Israel-Wilson base that has $V_{-}$unchanged and $V_{+}$given by (5.19). The spectral flow transformation preserves the regularity of the solution and replaces the supertube by a fluxed D6 brane, which is also perfectly regular considered either as either a six-dimensional or as a five-dimensional bubbled geometry. Hence, one obtains the smooth D6- $\overline{\mathrm{D} 6}$ solution with non-trivial flux described above. ${ }^{12}$

One can take this procedure further, and consider two or three different types of supertube in a GH geometry of the opposite orientation. Unlike the single supertube, this solution is no longer BPS, as the holonomy of the base metric is inconsistent with the supersymmetry projections associated with all the supertubes (the solution has three D2 and one $\overline{\mathrm{D} 6}$ charge).

If one now makes several spectral flows to convert each species of supertube to fluxes supported by geometry, the result must be regular for exactly the same reason that the BPS supertubes produce regular five-dimensional geometries after spectral flow: The almostBPS supertubes are locally identical to BPS supertubes and so the spectral flow cannot generate singularities. The result of such a multiple spectral flow must therefore be a completely regular, non-BPS geometry with fluxes in five dimensions. We expect that these solutions will go well beyond the Israel-Wilson class: Indeed, the metric coefficients of the base will generically involve products of more than two functions. We also expect this method to yield large classes of smooth horizonless non-BPS scaling solutions, which will be instrumental in extending the fuzball proposal to non-BPS extremal black holes.

\section{Conclusion}

We have shown that the equations of motion for five-dimensional ungauged supergravity coupled to three U(1) gauge fields can be reduced to a linear system using a floating-brane

\footnotetext{
${ }^{11}$ Essentially because the supertube only has two D2 charges, that are mutually-BPS with respect to a D6 brane irrespective of its orientation.

${ }^{12}$ It is worth commenting on how much supersymmetry this solution preserves. On one hand, we have obtained this solution by spectral flow from a supersymmetric solution. Since spectral flow is a combination of coordinate transformations and dualities, one would expect the resulting solution to still be supersymmetric. On the other hand, the resulting solution has an Israel-Wilson base, and, as proved in [17, 18], such solutions should not be supersymmetric, since all supersymmetric solutions must have a Hyper-Kähler base. The resolution of the puzzle is a generalization of that described in [54]: For these very special solutions the warp factors and angular momentum vector are such that if one makes a coordinate transformation of the type $\psi \rightarrow \psi+\alpha t$, and rewrites the metric as a time fibration over a four-dimensional base, this base space can be made hyper-Kähler. Hence this particular floating-brane solution is secretly BPS.
} 
Ansatz in which M2 branes feel no force, and hence the warp factors and the Maxwell electric potentials are equal. Upon making a simplifying assumption we have obtained a new class of non-BPS solutions, that are constructed starting not from a hyper-Kähler base (like the BPS and almost-BPS solutions) but from a much more general EinsteinMaxwell Euclidean electrovac solution, and solving a new linear system of equations for the warp factors and magnetic potentials. These "simplified floating brane" solutions are much more general than both the BPS and almost-BPS solutions, and reduce to them when the electrovac Maxwell fields are self- or anti-self-dual, and the base becomes Ricciflat. We have also noted that this implies that the BPS and "almost-BPS" equations yield full solutions of the supergravity equations of motion not only when the base is hyperKähler (as previously thought) but also when the base is Ricci-flat. A few such solutions are presented in [23].

The floating brane Ansatz requires that the warp factors and the electric potentials are equal. Not only does this result in no force upon appropriate brane probes but it also means that the mass of our solutions will be linear in their M2 brane charges. Hence, the non-BPS solutions that result from our Ansatz will naturally describe single or multiple extremal black holes, as well as smooth horizonless solutions that have the same charges and mass as extremal black holes. One can think about these solutions as having D-brane components that locally preserve some supersymmetries but whose supersymmetries are either globally incompatible with one another, or are broken by the gravitational background. Thus, we cannot hope to use this approach to obtain completely general non-extremal solutions, but only interesting sub-classes (like the Running-Bolt [23]) in which the mass is linear in the electric charges.

We have illustrated our method by finding a new two-center solution that has as a base space an Israel-Wilson metric, and that describes a D6-D4-D2-D0 black hole in the background of an anti-D6 brane. We have also shown that spectral flow can be used to map some of the new solutions into previously-known "almost-BPS" solutions.

While we have presented the material here in what seems to us as the natural expository order, we initially discovered examples of Israel-Wilson solutions by considering spectral flows of non-BPS solutions on GH spaces. This closely parallels the history of the discovery of the importance of ambi-polar base spaces for the constructions of BPS horizonless bubbling solutions; the crucial first examples of such metrics were obtained by spectral flow in $[39,40,56]$ and then greatly generalized and more deeply understood via geometric transitions in $[57,58]$. It is thus evident that spectral flow is a very powerful tool in suggesting completely new classes of physically-interesting solutions that can then be further generalized. In this paper we have only really exploited a single spectral flow and it is therefore very natural to continue exploring the more-complicated solutions generated by two or three spectral flows. It would be very interesting to see if one could find an Ansatz (similar to the floating-brane Ansatz used here) that describes such solutions, and if they could still be obtained using a linear procedure.

We have also obtained a smooth two-center non-BPS solution that describes a D6 and an anti-D6 brane kept in equilibrium by flux on the two-cycle between them. We would like to note that there exists a solution where such branes are kept in equilibrium by a 
background magnetic field [65, 66]. Furthermore, the non-BPS running Kerr-Taub-Bolt solutions recently constructed in [23] can be thought of as describing a D6 and an anti-D6 brane kept in equilibrium by both flux on the bolt and background magnetic field. It is quite likely that the floating-brane solutions constructed using Euclidean Reissner-Nordstrom electrovac base spaces [25] will have a similar interpretation. It would be interesting to explore all the possible ways of constructing a non-BPS D6 - anti D6 system in equilibrium, and to see whether one might be able to build two distinct supergravity solutions with the same brane interpretation.

We have made some conjectures as to the form of the solutions that will arise from multiple spectral flows, and they certainly will go beyond the Israel-Wilson electrovac backgrounds. We also expect that such solutions will describe even more general black holes and horizonless solutions. On the other hand, putting these solutions in the sixdimensional form that makes spectral flow into a mere coordinate transformation makes it clear that a spectral flow will always preserve the same flat $\mathbb{R}^{3}$ section of the base. Spectral flow simply does not affect this $\mathbb{R}^{3}$ structure and so even if the solutions that come out after multiple spectral flows are more general, the spatial metric will still have the form of $\mathrm{U}(1)^{2}$ fibrations over an $\mathbb{R}^{3}$ base. At first glance it appears that these solutions will not be general enough to describe all extremal black holes and black rings. For example, neither the extremal overspinning Rasheed-Larsen black hole [43-45] nor the extremal non-BPS three-charge black ring [46-48] can be written as fibrations over a flat $\mathbb{R}^{3}$ base. However, it is likely they will describe very large and non-trivial classes of non-BPS solutions, that will yield interesting physics.

\section{Acknowledgments}

We would like to thank N. Bobev, K. Goldstein, S. Katmadas and G. Gibbons for valuable discussions. NPW is grateful to the IPhT(SPhT), CEA-Saclay for hospitality while this work was done. The work of IB, CR and SG was supported in part by the DSM CEA-Saclay, by the ANR grants BLAN 06-3-137168 and 08-JCJC-0001-01, and by the Marie Curie IRG 046430. The work of NPW was supported in part by DOE grant DE-FG03-84ER-40168.

Open Access. This article is distributed under the terms of the Creative Commons Attribution Noncommercial License which permits any noncommercial use, distribution, and reproduction in any medium, provided the original author(s) and source are credited.

\section{References}

[1] V. Belinski and E. Verdaguer, Gravitational solitons, Cambridge University Press, Cambridge U.K. (2001) [SPIRES].

[2] S. Chiossi and S. Salamon, The intrinsic torsion of $\mathrm{SU}(3)$ and $\mathrm{G}_{2}$ structures, in Differential Geometry, Valencia 2001, World Scientific Publishing, Singapore (2002), pg. 115-133 [math/0202282] [SPIRES].

[3] J.P. Gauntlett and S. Pakis, The geometry of D = 11 Killing spinors, JHEP 04 (2003) 039 [hep-th/0212008] [SPIRES]. 
[4] P. Kaste, R. Minasian, M. Petrini and A. Tomasiello, Nontrivial RR two-form field strength and SU(3)-structure, Fortsch. Phys. 51 (2003) 764 [hep-th/0301063] [SPIRES].

[5] C.N. Gowdigere, D. Nemeschansky and N.P. Warner, Supersymmetric solutions with fluxes from algebraic Killing spinors, Adv. Theor. Math. Phys. 7 (2004) 787 [hep-th/0306097] [SPIRES].

[6] A.A. Pomeransky, Complete integrability of higher-dimensional Einstein equations with additional symmetry and rotating black holes, Phys. Rev. D 73 (2006) 044004 [hep-th/0507250] [SPIRES].

[7] A.A. Pomeransky and R.A. Sen'kov, Black ring with two angular momenta, hep-th/0612005 [SPIRES].

[8] H. Elvang and P. Figueras, Black Saturn, JHEP 05 (2007) 050 [hep-th/0701035] [SPIRES].

[9] S. Giusto and A. Saxena, Stationary axisymmetric solutions of five dimensional gravity, Class. Quant. Grav. 24 (2007) 4269 [arXiv:0705.4484] [SPIRES].

[10] J. Ford, S. Giusto, A. Peet and A. Saxena, Reduction without reduction: Adding KK-monopoles to five dimensional stationary axisymmetric solutions, Class. Quant. Grav. 25 (2008) 075014 [arXiv:0708.3823] [SPIRES].

[11] J. Evslin and C. Krishnan, The Black Di-Ring: An Inverse Scattering Construction, Class. Quant. Grav. 26 (2009) 125018 [arXiv:0706.1231] [SPIRES].

[12] J. Evslin and C. Krishnan, Metastable Black Saturns, JHEP 09 (2008) 003 [arXiv:0804.4575] [SPIRES].

[13] J. Evslin, Geometric Engineering 5D Black Holes with Rod Diagrams, JHEP 09 (2008) 004 [arXiv: 0806.3389] [SPIRES].

[14] R. Emparan, T. Harmark, V. Niarchos and N.A. Obers, Blackfolds, Phys. Rev. Lett. 102 (2009) 191301 [arXiv:0902.0427] [SPIRES].

[15] R. Emparan, T. Harmark, V. Niarchos and N.A. Obers, Essentials of Blackfold Dynamics, arXiv:0910.1601 [SPIRES].

[16] R. Emparan and H.S. Reall, Black Holes in Higher Dimensions, Living Rev. Rel. 11 (2008) 6 [arXiv:0801.3471] [SPIRES].

[17] J.P. Gauntlett, J.B. Gutowski, C.M. Hull, S. Pakis and H.S. Reall, All supersymmetric solutions of minimal supergravity in five dimensions, Class. Quant. Grav. 20 (2003) 4587 [hep-th/0209114] [SPIRES].

[18] J.B. Gutowski and H.S. Reall, General supersymmetric AdS $S_{5}$ black holes, JHEP 04 (2004) 048 [hep-th/0401129] [SPIRES].

[19] I. Bena and N.P. Warner, One ring to rule them all ... and in the darkness bind them?, Adv. Theor. Math. Phys. 9 (2005) 667 [hep-th/0408106] [SPIRES].

[20] K. Goldstein and S. Katmadas, Almost BPS black holes, JHEP 05 (2009) 058 [arXiv: 0812.4183] [SPIRES].

[21] I. Bena, G. Dall'Agata, S. Giusto, C. Ruef and N.P. Warner, Non-BPS Black Rings and Black Holes in Taub-NUT, JHEP 06 (2009) 015 [arXiv:0902.4526] [SPIRES].

[22] I. Bena, S. Giusto, C. Ruef and N.P. Warner, Multi-Center non-BPS Black Holes - the Solution, JHEP 11 (2009) 032 [arXiv:0908.2121] [SPIRES]. 
[23] I. Bena, S. Giusto, C. Ruef and N.P. Warner, A (Running) Bolt for New Reasons, JHEP 11 (2009) 089 [arXiv: 0909.2559] [SPIRES].

[24] P. Galli and J. Perz, Non-supersymmetric extremal multicenter black holes with superpotentials, arXiv:0909.5185 [SPIRES].

[25] N. Bobev and C. Ruef, The Nuts and Bolts of Einstein-Maxwell Solutions, JHEP 01 (2010) 124 [arXiv:0912.0010] [SPIRES].

[26] J. Camps, R. Emparan, P. Figueras, S. Giusto and A. Saxena, Black Rings in Taub-NUT and D0-D6 interactions, JHEP 02 (2009) 021 [arXiv:0811.2088] [SPIRES].

[27] I. Bena, N. Bobev and N.P. Warner, Spectral Flow and the Spectrum of Multi-Center Solutions, Phys. Rev. D 77 (2008) 125025 [arXiv:0803.1203] [SPIRES].

[28] G. Lopes Cardoso, A. Ceresole, G. Dall'Agata, J.M. Oberreuter and J. Perz, First-order flow equations for extremal black holes in very special geometry, JHEP 10 (2007) 063 [arXiv:0706.3373] [SPIRES].

[29] E.G. Gimon, F. Larsen and J. Simon, Black Holes in Supergravity: the non-BPS Branch, JHEP 01 (2008) 040 [arXiv:0710.4967] [SPIRES].

[30] K. Hotta and T. Kubota, Exact Solutions and the Attractor Mechanism in Non-BPS Black Holes, Prog. Theor. Phys. 118 (2007) 969 [arXiv:0707.4554] [SPIRES].

[31] I. Bena, Splitting hairs of the three charge black hole, Phys. Rev. D 70 (2004) 105018 [hep-th/0404073] [SPIRES].

[32] H. Elvang, R. Emparan, D. Mateos and H.S. Reall, Supersymmetric 4 D rotating black holes from 5D black rings, JHEP 08 (2005) 042 [hep-th/0504125] [SPIRES].

[33] D. Gaiotto, A. Strominger and X. Yin, 5D black rings and 4D black holes, JHEP 02 (2006) 023 [hep-th/0504126] [SPIRES].

[34] I. Bena, P. Kraus and N.P. Warner, Black rings in Taub-NUT, Phys. Rev. D 72 (2005) 084019 [hep-th/0504142] [SPIRES].

[35] V. Balasubramanian, J. de Boer, E. Keski-Vakkuri and S.F. Ross, Supersymmetric conical defects: Towards a string theoretic description of black hole formation, Phys. Rev. D 64 (2001) 064011 [hep-th/0011217] [SPIRES].

[36] J.M. Maldacena and L. Maoz, De-singularization by rotation, JHEP 12 (2002) 055 [hep-th/0012025] [SPIRES].

[37] O. Lunin, Adding momentum to D1-D5 system, JHEP 04 (2004) 054 [hep-th/0404006] [SPIRES].

[38] J. Ford, S. Giusto and A. Saxena, A class of BPS time-dependent 3-charge microstates from spectral flow, Nucl. Phys. B 790 (2008) 258 [hep-th/0612227] [SPIRES].

[39] S. Giusto, S.D. Mathur and A. Saxena, Dual geometries for a set of 3-charge microstates, Nucl. Phys. B 701 (2004) 357 [hep-th/0405017] [SPIRES].

[40] S. Giusto, S.D. Mathur and A. Saxena, 3-charge geometries and their CFT duals, Nucl. Phys. B $\mathbf{7 1 0}$ (2005) 425 [hep-th/0406103] [SPIRES].

[41] J.B. Gutowski, D. Martelli and H.S. Reall, All supersymmetric solutions of minimal supergravity in six dimensions, Class. Quant. Grav. 20 (2003) 5049 [hep-th/0306235] [SPIRES]. 
[42] M. Cariglia and O.A.P. Mac Conamhna, The general form of supersymmetric solutions of $N=(1,0) \mathrm{U}(1)$ and $\mathrm{SU}(2)$ gauged supergravities in six dimensions, Class. Quant. Grav. 21 (2004) 3171 [hep-th/0402055] [SPIRES].

[43] D. Rasheed, The Rotating dyonic black holes of Kaluza-Klein theory, Nucl. Phys. B 454 (1995) 379 [hep-th/9505038] [SPIRES].

[44] T. Matos and C. Mora, Stationary dilatons with arbitrary electromagnetic field, Class. Quant. Grav. 14 (1997) 2331 [hep-th/9610013] [SPIRES].

[45] F. Larsen, Rotating Kaluza-Klein black holes, Nucl. Phys. B 575 (2000) 211 [hep-th/9909102] [SPIRES].

[46] R. Emparan, Rotating circular strings and infinite non-uniqueness of black rings, JHEP 03 (2004) 064 [hep-th/0402149] [SPIRES].

[47] H. Elvang, R. Emparan and P. Figueras, Non-supersymmetric black rings as thermally excited supertubes, JHEP 02 (2005) 031 [hep-th/0412130] [SPIRES].

[48] R. Emparan, Exact Microscopic Entropy of Non-Supersymmetric Extremal Black Rings, Class. Quant. Grav. 25 (2008) 175005 [arXiv:0803.1801] [SPIRES].

[49] V. Jejjala, O. Madden, S.F. Ross and G. Titchener, Non-supersymmetric smooth geometries and D1-D5-P bound states, Phys. Rev. D 71 (2005) 124030 [hep-th/0504181] [SPIRES].

[50] V. Cardoso, O.J.C. Dias, J.L. Hovdebo and R.C. Myers, Instability of non-supersymmetric smooth geometries, Phys. Rev. D 73 (2006) 064031 [hep-th/0512277] [SPIRES].

[51] W. Israel and G.A. Wilson, A class of stationary electromagnetic vacuum fields, J. Math. Phys. 13 (1972) 865 [SPIRES].

[52] B. Whitt, Israel-Wilson metrics, Ann. Phys. 161 (1985) 241 [SPIRES].

[53] A.L. Yuille, Israel-Wilson Metrics in the euclidean regime, Class. Quant. Grav. 4 (1987) 1409 [SPIRES].

[54] M. Dunajski and S.A. Hartnoll, Einstein-Maxwell gravitational instantons and five dimensional solitonic strings, Class. Quant. Grav. 24 (2007) 1841 [hep-th/0610261] [SPIRES].

[55] I. Bena, N. Bobev, C. Ruef and N.P. Warner, Supertubes in Bubbling Backgrounds: Born-Infeld Meets Supergravity, JHEP 07 (2009) 106 [arXiv:0812.2942] [SPIRES].

[56] S. Giusto and S.D. Mathur, Geometry of D1-D5-P bound states, Nucl. Phys. B 729 (2005) 203 [hep-th/0409067] [SPIRES].

[57] I. Bena and N.P. Warner, Bubbling supertubes and foaming black holes, Phys. Rev. D 74 (2006) 066001 [hep-th/0505166] [SPIRES].

[58] P. Berglund, E.G. Gimon and T.S. Levi, Supergravity microstates for BPS black holes and black rings, JHEP 06 (2006) 007 [hep-th/0505167] [SPIRES].

[59] S.D. Mathur, The fuzzball proposal for black holes: An elementary review, Fortsch. Phys. 53 (2005) 793 [hep-th/0502050] [SPIRES].

[60] I. Bena and N.P. Warner, Black holes, black rings and their microstates, Lect. Notes Phys. 755 (2008) 1 [hep-th/0701216] [SPIRES].

[61] K. Skenderis and M. Taylor, The fuzzball proposal for black holes, Phys. Rept. 467 (2008) 117 [arXiv:0804.0552] [SPIRES]. 
[62] V. Balasubramanian, J. de Boer, S. El-Showk and I. Messamah, Black Holes as Effective Geometries, Class. Quant. Grav. 25 (2008) 214004 [arXiv:0811.0263] [SPIRES].

[63] O. Lunin and S.D. Mathur, AdS/CFT duality and the black hole information paradox, Nucl. Phys. B 623 (2002) 342 [hep-th/0109154] [SPIRES].

[64] O. Lunin, J.M. Maldacena and L. Maoz, Gravity solutions for the D1-D5 system with angular momentum, hep-th/0212210 [SPIRES].

[65] D.J. Gross and M.J. Perry, Magnetic Monopoles in Kaluza-Klein Theories, Nucl. Phys. B 226 (1983) 29 [SPIRES].

[66] A. Sen, Strong coupling dynamics of branes from M-theory, JHEP 10 (1997) 002 [hep-th/9708002] [SPIRES]. 